\title{
Ionization Phenomena in a Gas-Particle Plasma
}

\author{
Edward G. Gibson* \\ Daniel and Florence Guggenheim Jet Propulsion Center, \\ California Institute of Technology, Pasadena, California \\ (Received 15 December 1965; final manuseript received 1 September 1966)
}

\begin{abstract}
Particles in a plasma can appreciably change the electron density from the value it would assume if the particles were not present. The case of pure particle ionization, in which there is only thermionic emission from the particles and no gas ionization, is first considered. It is established that the potential and the charge distributions can be divided into a strong shielding regime, in which most of the free electrons are packed close to the particle surfaces in regions of high potential, and its direct opposite, a weak shielding regime. In both regimes, the free-electron content of the plasma is most readily altered by variations in the particle size, rather than in the work function or particle temperature. The suppression of one form of ionization by the other when both particle and gas contribution to the electron density are comparable is next investigated. In the case of gaseous ionization enhancement it is shown that, if the thermionically emitting particles are hotter than the gas, the electron temperature will also be higher than that of the gas and the gaseous ionization thereby enhanced. Lastly, it is shown that in some transient situations, the particles are able to control the time rate of change of the electron density.
\end{abstract}

\section{INTRODUCTION}

$\mathrm{T}$ $\mathrm{HE}$ interest which has been generated in recent years in the physics of plasmas is due mainly to the ability of a plasma to interact with an electromagnetic field. The strength of this interaction is largely determined by the magnitude of the electron density in the plasma. Thus, any phenomenon which could appreciably alter this density is worth considerable attention. Variations in the temperature of the entire system, variations in just the electron temperature (produced by the application of an electromagnetic field), or changes in the effective ionization potential of the plasma (produced by the addition of an alkali metal vapor or a high electron affinity material) are all well-known methods which are able to produce relatively large changes in the electron density.

Recently, attention has been directed towards ionization phenomena arising in plasmas that are composed of two states of matter, gas-solid or gasliquid, in which the nongaseous state is in the form of small particles, $10^{-6}$ to $10^{-3} \mathrm{~cm}$ in size and may or may not be thermionically emitting. In many situations, some in which the particles arise naturally and others in which they are intentionally added, the electron density can be considerably altered through the presence of these particles.

Some examples of gas-particle plasmas which have been investigated and where the interaction with an electromagnetic field is, or could be, of large importance are:

* Present address: Manned Spacecraft Center, National Aeronautics and Space Adminstration, Houston, Texas.
(1) the combustion products of a solid rocket motor which contain alumina particles, ${ }^{1}$

(2) the combustion products of a fuel-rich hydrocarbon flame in which carbon particles have formed, ${ }^{2-5}$

(3) the plasma in the electrode section of a magnetohydrodynamic power generator where a fraction of the alkali metal vapor has condensed into liquid particles, ${ }^{\circ}$

(4) the wake of a re-entry body in which particles may exist or could be added.

When thermionically emitting particles are present in an ionized gas, there are many interesting interactions which can take place between the two types of ionization. In fact, there is a whole spectrum of problems to be associated with the two extreme cases of pure particle ionization, in which there is no gas ionization, and pure gas ionization, in which there is no thermionic emmission from the particles. The main objective of this investigation is to develop both qualitative and quantitative understanding of the various ionization phenomena which can occur in a gas-particle plasma. Of special interest are those phenomena in which the electron density of the plasma can be appreciably altered through

1 F. T. Smith and C. R. Gatz, in Progress in Astronautics and Aeronautics: Ionization in High Temperature Gases, K. E. Shuler, Ed. (Academic Press Inc., New York, 1963), p. 301.

2 K. E. Shuler and J. Weber, J. Chem. Phys. 22, 491 (1954).

${ }^{3}$ H. Einbinder, J. Chem. Phys. 26, 948 (1957).

${ }^{4}$ F. T. Smith, J. Chem. Phys. 28, 746 (1958).

- F. T. Smith, in Proceedings of the Third Conference on Carbon (Pergamon Press, Inc., London, 1959), p. 419.

J. M. Smith, AIAA J. 3, 648 (1965). 
the presence of the particles. ${ }^{7}$ In order that this investigation can be limited to a tractable length and scope, the gas-particle plasma is treated as a closed system. That is, ionization phenomena which arise directly from outside influences are not considered. Interactions thus excluded include photoionization of the gas, photoelectric ionization of the particles, and enhanced gaseous ionization through electron heating with an externally applied electromagnetic field.

\section{EQUILIBRIUM PARTICLE IONIZATION}

In a steady-state gas-particle plasma, the potential and charge distributions are closely coupled to one another. Thus, in order to describe the properties of the plasma, these two distributions must be determined simultaneously. In the problem which is now to be solved, that of equilibrium particle ionization, this is done by finding the solution to Poisson's equation for a one-particle system in which the potential and charge distributions are related by the Boltzmann factor and the only source of electrons is thermionic emission from the particles. The understanding gained about the nature of the potential and charge distributions proves to be of considerable assistance in the analysis of other situations.

\section{A. Assumptions and Model}

The assumptions which define the model to be used in this problem are the following:

(1) Steady statc. This will almost always be a valid assumption for problems in which there is no gaseous ionization because the characteristic time for the redistribution of the thermionically emitted electrons is usually very much smaller than the characteristic times for other changes in the system.

(2) Negligible gas ionization.

(3) Uniform particle properties. The assumption is made that all of the parameters which determine the degree of particle ionization in the plasma are the same for all particles. These parameters are: the temperature of the system $T$, the particle radius $a$, and the electron density at the surface of a particle due to thermionic emission $n_{e a}$.

(4) Uniform particle ionization. It is also assumed that the net number of electrons emitted from an initially uncharged particle $z$ is the same for all particles. The validity of this assumption is deter-

7 The work which is reported in this paper is abstracted from a Ph.D. thesis, California Institute of Technology (1964). A much fuller treatment of ionization phenomena in a gasparticle plasma is to be found in this reference. mined later when the results found here are compared with those which are found by taking into account the statistical distribution of particles over the available ionized states.

(5) Spherical symmetry. The assumption is made that the particles are spherical and that the surrounding potential and charge distributions are spherically symmetric. In a large number of systems, the particles are nearly spherical because they are either in or directly formed from the liquid state (the spherical nature of the particles in two particular systems is directly exhibited in the photographs of Millikan ${ }^{8}$ ).

The problem has now been reduced to the investigation of a one-particle system of inner radius $a$, the particle radius, and outer radius $b$, the radius which defines the volume that is allotted to each particle. That is, $4 / 3 \pi b^{3}=1 / N$, where $N$ is tho particle number density.

(6) Charge neutrality. It is assumed that the plasma is macroscopically neutral. Thus, the total charge inside the radius $b$ is zero and, by Gauss' law, the electric field at $r=b$ is also zero.

(7) Equilibrium. The system is assumed to be in thermodynamic equilibrium so that the potential and electron charge distributions are related by the Boltzmann factor. At the position radius $r$, the origin of which is at the center of the particle, the electron density is given by

$$
n_{e}(r)=n_{e b} \exp [e \phi(r) / k T],
$$

where $n_{e b}=n_{e}(b), \phi$ is the electrostatic potential, $k$ is the Boltzmann constant, and $\phi(b)$ has been set equal to zero.

(8) Electron density at a particle's surface. It is assumed that the electron density at the surface of a particle is known. When statistical mechanics is applied to the problem of thermionic emission from a conducting material, an emission law can be obtained. It is found that the emission from many materials can be described by an emission law of this form, over temperature increments large enough to be useful, but that the constants in the law must be obtained empirically. Thus, the electron density immediately outside of a thermionically emitting surface at equilibrium, $n_{E}$, is given by

$$
n_{E}=\kappa B T^{\frac{n}{2}} \exp (-e \Phi / k T),
$$

where $B=4.83 \times 10^{15} \mathrm{~cm}^{-3}{ }^{\circ} \mathrm{K}^{-\frac{3}{2}}$, the theoretical value, $\kappa$ is an adjustment coefficient, and $e \Phi$ is the thermionic work function. Both $\kappa$ and $e \Phi$ are found

${ }^{8}$ R. C. Millikan, J. Opt. Soc. Am. 51, 539 (1961). 
from experiment. ${ }^{9,10}$ Further assumptions which must be made in order to equate the electron density at the surface of a particle $n_{e a}$ to $n_{E}$ are: (a) The net loss of electrons from the conduction band in a particle is negligible. (b) The surface charge on a particle does not significantly change its emission properties. (c) The characteristic length of the potential field around the particle must be large with respect to the characteristic length of the longrange mirror-image potential of the work function in order for the definition of $n_{E}$, the electron density "immediately outside a surface," to be physically meaningful. This will require that the magnitude of the characteristic length of the field around the particle be larger than $10^{-7} \mathrm{~cm}$. (d) The changes which occur in the thermonic work function due to gas absorption on the particles can be predicted.

\section{B. Statement of the Problem and the Method of Solution}

For the one-particle system, the problem is now specified by Poisson's equation,

$$
\frac{1}{x^{2}} \frac{d}{d x}\left(x^{2} \frac{d y}{d x}\right)=\left(\frac{b}{\lambda_{D b}}\right)^{2} e^{y}
$$

and the boundary conditions $y=0$ and $d y / d x=0$ at $x=1$, where $y=e \phi / k T, x=r / b$, and $\lambda_{D b}=$ $\left[\epsilon_{0} k T / e^{2} n_{e b}\right]^{\frac{1}{2}}$, the Debye shielding distance at $r=b$. The solution to any given problem is defined by the two ratios $b / a$ and $b / \lambda_{D b}$. However, since it is $\lambda_{D a}$ which is known at the outset of the problem and $\lambda_{D a}=\lambda_{D b} \exp \left(-\frac{1}{2} y a\right)$, it is preferable to use $b / a$ and $b / \lambda_{D a}$ in specifying the interesting quantities which arise from the solution.

Equation (1) is a transcendental equation of the Emden type ${ }^{11}$ and no analytical solutions are known to the author. An IBM 7090 was therefore used to integrate this equation and to obtain a family of curves for 20 different values of $b / \lambda_{D b}$ between 0.1 and 2.0. The integrations were run between the limits of $1 \leq b / r \leq 10^{3}$ or $0 \leq y \leq 18.5$, depending upon which upper limit was reached first.

\section{Solution}

By examining the nature of the solution, it is possible to divide this shielding problem into two

${ }^{9}$ J. D. Cobine, Gaseous Conductors (McGraw-Hill Book Company, Inc., New York, 1958), p. 109.

${ }_{10}$ W. B. Nottingham, in Handbuch der Physik, S. Flügge, Ed. (Springer-Verlag, Berlin, 1956), Vol. XXI, p. 1. Many other sources of empirical values of $\kappa$ and $e \Phi$ are given in this reference.

${ }_{11}$ H. T. Davis, Introduction to Non-Linear Differential and Integral Equations (United States Government Printing Office, Washington, D. C., 1960), p. 381. characteristic regimes and find an approximate solution which is very good in one of them.

\section{Weak Shielding Regime}

The manner in which an approximate solution arises can be understood by rewriting and examining Eq. (1).

$$
\frac{d^{2} y}{d x^{2}}=\left(\frac{b}{\lambda_{D b}}\right)^{2} e^{y}-\frac{2}{x} \frac{d y}{d x}
$$

The relative magnitude of the two terms on the right-hand side of Eq. (2) will determine the nature of the solution. It is therefore informative to examine the ratio

$$
R=-\frac{2}{x} \frac{d y}{d x} /\left(\frac{b}{\lambda_{D b}}\right)^{2} e^{y} .
$$

When $r / b$ is close to $1, R \simeq 0$ and $e^{y} \simeq 1$. As $r / b$ decreases, $R$ becomes very much larger than 1 before $e^{y}$ changes appreciably from 1 for the range of $b / \lambda_{D b}$ investigated. Thus, an approximate solution to Eq. (1) is found by setting $e^{y}=1$ and integrating. The result is

$$
y=\frac{1}{3}\left(b / \lambda_{D b}\right)^{2}\left[b / r-\frac{3}{2}+\frac{1}{2}(r / b)^{2}\right] .
$$

As $r / b$ decreases further, $R$ continues to increase until the exponential behavior of the solution becomes significant and it then decreases and passes again through unity. The above solution is a good approximation up to this point. It should be noted that this approximation does not restrict $y$ to values which are small with respect to unity.

The numerical solutions to Eq. (1) are presented in Fig. 1 as a plot of $y$, normalized by $\frac{x}{3}\left(b / \lambda_{D b}\right)^{2}$ vs $b / r$. It is seen that the curves which correspond to the exact solutions coincide with the function $\left[(b / r)-\frac{3}{2}+\frac{1}{2}(r / b)^{2}\right]$, the curve for the approximate solution, up to limiting values of $b / r$.

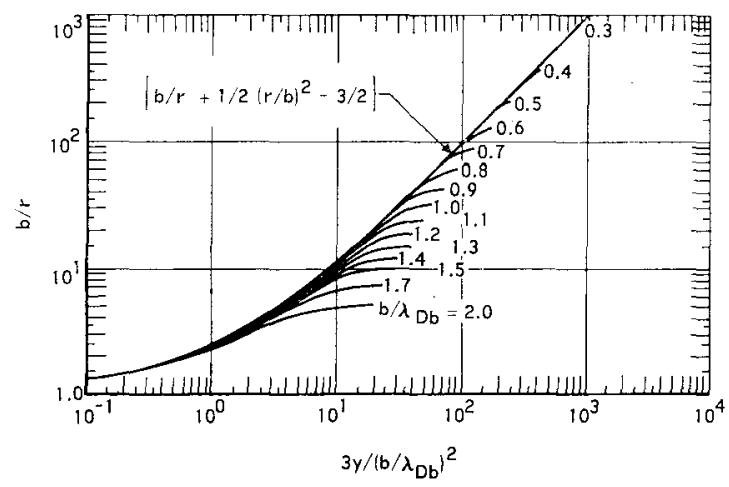

FIG. 1. Numerical solution to Poisson's equation normalized by $\frac{1}{3}\left(b / \lambda_{D b}\right)^{2}$. 


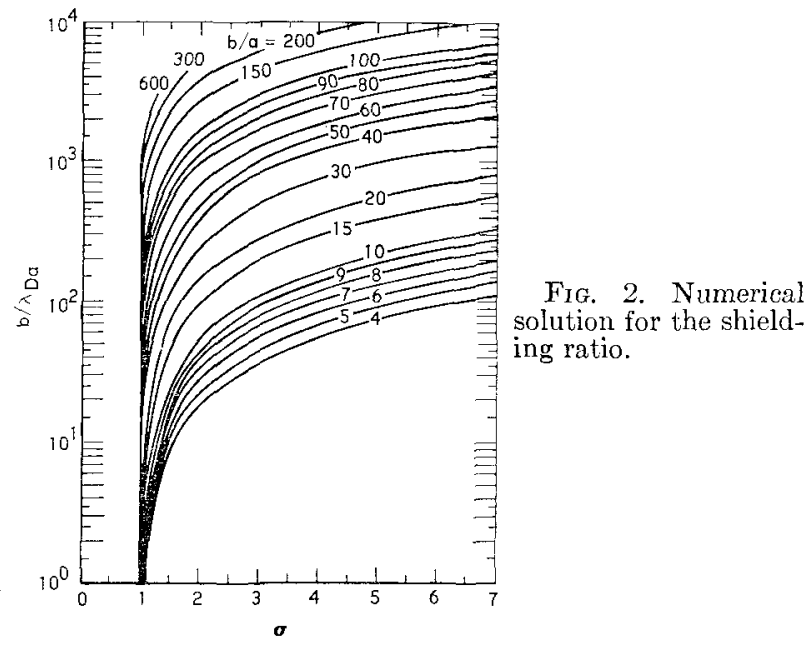

Physically, the approximate solution can be derived from the concept of a nearly constant density sea of electrons around the particles. In moving in toward a particle from $r=b$, the major fraction of the total number of emitted electrons is passed before the electron density has changed appreciably, because of the geometrical or $(r / b)^{3}$ effect. When the electron density is no longer constant, the field is determined almost entircly by the large positive charge on the particle. For $b / r \gg 1$, this is the dominant effect and $\phi \sim z / r$.

In the weak shielding approximation, the nondimensional potential of the particle $y_{a}$ and the electron density of the plasma $n_{e b}$ are readily determined from

$$
\begin{array}{r}
y_{a} e^{y_{a}}=\frac{1}{3}\left(b / \lambda_{D a}\right)^{2}\left[(b / a)-\frac{3}{2}+\frac{1}{2}(a / b)^{2}\right], \\
\left(n_{e a} / n_{e b}\right) \ln \left(n_{e a} / n_{e b}\right) \\
=\left(e^{2} n_{e a} / 4 \pi \epsilon_{0} a N k T\right)\left[1-\frac{3}{2}(a / b)+\frac{1}{2}(a / b)^{3}\right] . \\
\text { 2. Strong Shielding Regime }
\end{array}
$$

\section{Strong Shielding Regime}

' 1 'he weak shielding approximation is no longer valid when the effect of the one-dimensional shielding is larger than that of the three-dimensional geometry and a significant fraction of the total number of emitted electrons are located close to the particle. When a large fraction of the total number of emitted electrons are close to the particle, the divergence of the electric field will be appreciable $(R \ll 1)$ and the shielding will be relatively strong.

This physical interpretation suggests that a quantity which can be used to differentiate the strong and the weak shielding regimes is the ratio $\sigma=\vec{n}_{e} / n_{e b}$ where $\bar{n}_{\theta}$ is the mean electron density defined by $\bar{n}_{a}=z N$. When this shielding ratio $\sigma$ is close to unity, most of the electrons are in regions of nearly zero potential and the problem is characterized by weak shielding. When $\sigma$ is much larger than unity, the reverse is true. A plot of $\sigma$ as a function of $b / a$ and $b / \lambda_{D a}$ is given in Fig. 2 .

From Fig. 3 , it is seen that the divisions between the two shielding regimes defined by $R$ and $\sigma$ are esscntially the same. Also, the line which divides the two regimes by the condition $R=1$ can be approximated by a straight line of unit slope. This leads to the criterion that the weak shielding approximation will be valid if $a / \lambda_{D a}<5$, a criterion which makes quantitative that which would be expected on an intuitive basis. That is, if $\lambda_{D a} \ll a$, it is to be expected that the potential distribution close to the particle will be that of a one-dimensional sheath. If $a \ll \lambda_{D a}$, the potential distribution should be dominated by the $1 / r$ field. A second criterion for the validity of the weak shielding approximation, which can be established using Gauss' law and the requirement that $R>1$, is $z>2 \pi a^{3} n_{c a}$.

Plots of $y_{a}$ and $\vec{n}_{e} / n_{e a}$ as functions of $b / a$ and $b / \lambda_{D a}$, which cover both shielding regimes, are given in Ref. 7 .

\section{Comparison with the Results of the Statistical Method}

At the beginning of the equilibrium particle ionization problem, it was explicitly assumed that the particle ionization was uniform. However, in an actual system, there will be a distribution of the particles over the possible states of ionization even though the particle parameters $(a, T$, and $e \Phi)$ are uniform. It is instructive, therefore, to see what effect this distribution has on the detcrmination of $\bar{n}_{e}$. Also, when the majority of particles are not ionized many times, it is initially anticipated that the assumption of charge continuity breaks down and that the statistical approach is the one which must be used.

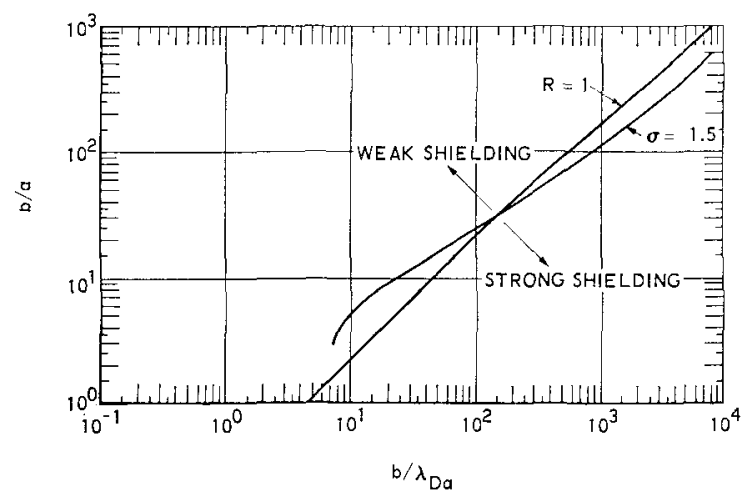

FIG. 3. Division between the weak and strong shielding regimes in the $\left(b / a, b / \lambda_{D a}\right)$ plane. 
A major difficulty encountered in the statistical approach is that the ionization potential for each degree of ionization must be known. If it is assumed that each electron which is external to a particle is at zero potential (weak shielding regime), these potentials are easily calculated. However, in some cases, a large fraction of the emitted electrons are not at zero potential (strong shielding regime). This second situation is analogous to a multipleionized atom in which there are as many or more electrons in the excited states than there are in the ionized states. Thus, if the statistical approach is to be used in a rigorous way to calculate the electron density in a system of thermionically emitting particles, the statistical weights corresponding to the continuum of energy levels must first be known. The difficulty which is encountered here is traced directly back to the coupling which exists between the potential and the charge distributions.

In their treatments of the problem, Einbinder ${ }^{3}$ and $\operatorname{Smith}^{4,5}$ have assumed that all of the emitted electrons are at zero potential. In contrast to Einbinder, however, Smith has taken into account the fact that the particles undergo electron attachment, as well as electron emission, and thereby produce negative degrees of ionization. By writing down the cquilibrium constants for each succeeding degree of ionization, he has found that the relation for the distribution of particles over the ionized states is a Gaussian function which can be written as

$$
N_{l} / N_{0}=e^{\frac{1}{2}(z y a)} e^{-\frac{1}{2}[(l-z) / \delta l]},
$$

where $N$ is the number density of particles which are ionized $l$ times and the values of $y_{a}$ and $z$ correspond to the assumption of uniform ionization; $y_{a}=\ln \left(n_{E} / n_{e b}\right)$ and $z=y_{a} C k T / e^{2}$. Here, $C$ is the electrostatic capacitance of a particle and $\delta l$ is the width of the distribution which is given by $\delta l=$ $\left(z / y_{a}\right)^{\frac{1}{2}}$.

The significance of this result, in relation to that which has been obtained by the uniform ionization assumption, is that: (1). The peak of the distribution is at $l=z$ and the relative width is $\delta l / z=\left(y_{a} z\right)^{-\frac{1}{2}}$. Thus, when $\left(y_{a} z\right)^{\frac{1}{2}} \gg 1$, the ionization is essentially uniform. (2) Smith has stated that the mean value of $l$ differs only negligibly from $z$, the uniform ionization value, as long as $e^{2} /(2 C k T)<3$. That is, as long as $\delta l>0.4$, the uniform ionization and continuous charge approximations will lead to the correct value of $n_{e b}$, even if $z$ is of the same magnitude as or less than unity. For spherical particles with $a / b \ll 1$ and at a temperature of $2000^{\circ} \mathrm{K}$, the above requirement means that $a$ must be larger than $14 \AA$, a requirement that, practically speaking, is not very restrictive.

\section{Dependence of the Electron Density on the Initial Parameters}

Two primary objectives of an investigation of the ionization in a gas-particle plasma should be first, to arrive at a prediction of the electron density, and second, to obtain an estimate of the extent to which this electron density can be controlled. Hence, it is profitable to determine the dependence of the electron density on the parameters which define the solution for the equilibrium particle ionization problem.

\section{Strong Shielding Regime}

In the strong shielding regime, there are two electron densities of interest. One is the "free electron" density, or the density outside the charge sheath around each particle $n_{e b}$. These electrons are not bound close to a particle's surface by strong fields and are relatively free to move throughout the plasma. The second density of interest is $\bar{n}_{\theta}$, which is representative of the degree of particle ionization $\left(z=\bar{n}_{e} / N\right)$.

In the numerical investigation of the equilibrium particle ionization problem, it was found that once the strong shielding rogime was encountered, the electron charge distribution always adjusted itself so that the value of $\lambda_{D b}$ was of the same magnitude as $b$. This will place an upper limit on $n_{e b}$. The numerical analysis shows that in most cases the maximum value of $b / \lambda_{D b}$ is approximately 2. Thus, the corresponding upper limit on $n_{e b}$ is $n_{e b}^{(\max )}=$ $4 \epsilon_{0} k T /(e b)^{2}$. For $T=2900^{\circ} \mathrm{K}$ and $N=10^{8} \mathrm{~cm}^{-3}$, $n_{e b}^{(\max )}=3.1 \times 10^{11} \mathrm{~cm}^{-3^{\circ}}$. The only way in which $n_{e b}$ can be changed by large factors is by changing $N$. That is $n_{e b} \sim T N^{3}$ or, if the total nongaseous content is held fixed $\left(\mathrm{Na}^{3}=\right.$ const), $n_{e b} \sim T / a^{2}$.

If it is assumed that the situation of interest is well into the strong shiclding regime $(\sigma \gg 1)$, the value of $\bar{n}_{e}$ will approximately equal the number of electrons packed close to a particle in its sheath times the number density of particles. Thus, $\bar{n}_{e} \sim$ $a^{2} \lambda_{D a} n_{e a} N$ or, $\bar{n}_{\varepsilon} \sim a^{2} N\left(T n_{e a}\right)^{\frac{1}{2}}$. Again, if $N a^{3}$ is held constant, $n_{\varepsilon} \sim\left(T n_{e a}\right)^{\frac{1}{2}} / a$. Hence, unlike $n_{e b}$, $\bar{n}_{e}$ will be an exponential function of $T$ and $e \Phi$.

\section{Weak Shielding Regime}

In the majority of enginecring problems, the condition $a / \lambda_{D a}<5$ is satisfied and the weak shielding regime is therefore the one most frequently encountered. 
Since $\bar{n}_{\bullet} \simeq n_{e b}$, only the variations of $n_{e b}$ are directly studied. The relationships which define the value of $n_{e b}$ are the Boltzmann relation, the thermionic emission law, and the approximate solution for the particle potential. The parameters are taken to be $e \Phi, T, a$, and $N$. The sensitivity of $n_{e b}$ to changes in $e \Phi$ is analyzed first.

It is convenient to refer all variables to a refcrence state. Letting primes denote the reference state conditions, the dependent variables are $\nu=$ $n_{e b} / n_{e b}^{\prime}$ and $\eta=\phi_{a} / \phi_{a}^{\prime} ;$ the independent variable is $\omega=e \Phi / e \Phi^{\prime}$; and the normalized particle potential and work function are $y_{a}=e \phi_{a}^{\prime} / k T^{\prime}$ and $w=e \phi^{\prime} / k T^{\prime}$.

Using the three relationships which define $n_{e b}$, the following are obtained.

$$
\begin{gathered}
\eta=\nu, \\
\nu=1+\left(w / y_{a}\right)(1-\omega)-\left(1 / y_{a}\right) \ln \nu,
\end{gathered}
$$

and

$$
d \nu /\left.d \omega\right|_{\omega=1}=-w /\left(y_{a}+1\right) .
$$

These equations clearly demonstrate the manner in which the effects of shielding tend to dominate those of emission as the particle potential of the reference state is increased. When $y_{a}$ is less than or on the order of unity, the value of $n_{e b}$ changes in an exponential manner with the work function. However, when $y_{a}$ is of the same magnitude as $w\left(e \phi_{a}^{\prime} \approx e \Phi^{\prime}\right)$, the level of ionization is controlled by shielding effects and the relation between the level of ionization and the work function is not an exponential one but is nearly a linear one. It is clear that the reason for this is the changes in $\phi_{a}$ which accompany those of $n_{e a}$. Thus, errors which are made in the estimation of $e \Phi$ or changes made in the actual value of $e \Phi$ will not greatly affect the value of the electron density in the plasma.

In the same manner, the changes which occur in $n_{\varepsilon b}$ due to variations of $T, a$, and $N$ can be analyzed. When this is done, the following conclusions are drawn.

(1) When the particle potential $e \phi_{a}$ is much less than the work function $e \Phi$, the electron density is exponentially dependent on $e \Phi$ and $T$, being most sensitive to the fractional changes in $T$.

(2) When $e \phi_{a}$ is of the same magnitude as $e \Phi$, the electron density is almost linearly related to $e \Phi$ and $T$ and is still more sensitive to fractional changes in $T$.

(3) If $e \phi_{a}$ is much less than or approximately equal to $k T$, the electron density is very insensitive to changes in $a$ or $N$.
(4) If $e \phi_{a}$ is much larger than $k T$, the electron density is directly proportional to $a$ or $N$, but varies as $1 / a^{2}$ when $N a^{3}$ is held constant.

Thus, it is probable that the largest error made in predicting the value of $n_{e b}$ will be due to errors made in determining $a$, since the percentage error made in the estimation of $a$ is likely to be much larger than those made in the estimation of $e \Phi$ or $T$. Also, since it is a which can be most easily changed by large factors for a given mass fraction, it is the particle size which can most effectively be used in controlling the electron density of the plasma.

In Ref. 7 , the problem of a nonuniform distribution of particle size, in which the potential distribution around each particle falls into the weak shielding regime, is considered. The general result is that the electron density of the plasma can be calculated with negligible error introduced if the values of $a$ and $N$ are defined such that the arithmetical mean particle radius and the total nongaseous content are the same as in the actual system. It is also shown that if the gas and particle temperatures differ, the value of the electron density will still be close to the value it would assume if the temperature of the whole system were that of the particles.

\section{PARTICLE POTENTIAL AND FLUX RELATIONS}

Both equilibrium and nonequilibrium ionization phenomena in which gaseous ionization is of importance are next investigated. However, before any special cases are considered, additional relations must be developed.

\section{A. Particle Potential}

The approximate solution for the potential distribution, which was found to be valid in the weak shielding regime in the particle ionization problem, is assumed to be valid here. That is, the physical concept of a nearly constant density sea of charge with abrupt changes occurring close to the particles is still applicable. However, rather than the electron density at $r=b$ being the important quantity in determining the charge on a particle, it is the net charge density at $r=b, e\left(n_{i b}-n_{e b}\right)$, where $n_{i b}$ is the ion density at $r=b$, which must be used. Since the plasma is assumed to be macroscopically neutral, the charge per particle is $e\left(n_{e b}-n_{i b}\right) / N$, which can be positive or negative. Thus, the particle potential in the weak shielding regime is

$$
e \phi_{a} / k T_{p}=\left[\left(n_{e b}-n_{i b}\right) / N\right] P\left(a, b, T_{P}\right),
$$


where

$P\left(a, b, T_{P}\right)=\left(e^{2} / 4 \pi \epsilon_{0} a k T_{p}\right)\left[1-\frac{3}{2}(a / b)+\frac{1}{2}(a / b)^{3}\right]$.

The criterion for the validity of this approximate solution again is that $a / \lambda_{D q}<5$. However, the species which has the sign opposite to that which resides on a particle must be used in determining $\lambda_{D a}$. This criterion is a conservative one since the presence of the second charged species tends to increase the characteristic length of the one-dimensional Debye sheath.

\section{B. Number and Energy Fluxes}

The number fluxes of electrons and ions moving to and from a particle must be known in order to investigate other steady and nonsteady situations; the steady-state requirement implies that the net flux of charge to a particle is zero and, in nonstcady processes, the rate of addition or removal of the charged species could be controlled by the magnitude of these fluxes. Also, in the cases in which knowledge of the electron temperature is of importance, the energy flux in the electrons emitted from and returning to a particle must also be known. It is necessary to discuss only the relations for the electrons since the relations for the ions are easily obtained by analogy.

The magnitude of the electron number and energy fluxes will be influenced by the size of the collisionless region which exists around a particle. When the electron mean free path $\lambda_{e}$ is much less than the particle radius, the motion of the electrons to and from a particle will be a diffusion process. However, when this region becomes large, the electron motion will be that of a three-dimensional free fall. A problem formulated in phase space which explores the effect on the fluxes as the free-fall region goes from a one-dimensional to a three-dimensional geometry has been considered in Ref. 7. In this problem, the velocity distribution of the electrons leaving the particle's surface was taken to be a half-Maxwellian and the collisionless region extended out to a radius $c\left(c \approx a+\lambda_{e}\right)$. The velocity distribution of the electrons, which move toward the particle from the electron gas at $r=c$, was assumed to also be a half-Maxwellian. The solution to this problem, which depends upon the sign of the particle potential, is now briefly discussed. However, only the results of the limiting case of $\lambda_{\varepsilon} \gg a$ are presented since it is this case which is most useful for later considerations. The results stated here are in agreement with those obtained by
Spitzer ${ }^{12,13}$ and Sodha, ${ }^{14,15}$ who implicitly assumed at the outset that $\lambda_{e} \gg a$.

When the particle potential is negative, the electron fluxes are

$$
\begin{gathered}
\Gamma_{n_{e}}^{+}=\frac{1}{4} n_{E}\left(8 k T_{p} / \pi m_{e} e^{\frac{1}{2}},\right. \\
\Gamma_{n_{e}}^{-}=\frac{1}{4} n_{e b}\left(8 k T_{e} / \pi m_{e}\right)^{\frac{1}{2}} e^{e \phi_{a} / k T_{e}}, \\
\Gamma_{H_{e}}^{+}=\Gamma_{n_{e}}^{+}\left(2 k T_{p}-e \phi_{a}\right), \\
\Gamma_{H_{e}}^{-}=\Gamma_{n_{o}}^{-}\left(2 k T_{e}-e \phi_{a}\right),
\end{gathered}
$$

where $\Gamma_{n_{0}}^{+}$is the number of electrons per unit time per unit area which leave the surface of the particle and escape into the electron gas, $\Gamma_{n_{8}}^{-}$is the corresponding flux for the electrons which reach the particle's surface from the electron gas, the subscript $H_{e}$ denotes the energy carried by the electron flux, and $T_{p}$ and $T_{e}$ are the temperatures of the particles and the electron gas, respectively. It is seen that for the negative particle potential case, the expression for the fluxes are the same as those which would be found from one-dimensional considerations.

When the particle potential is positive, an additional criterion which must be satisfied in order that the collisionless region can be considered large with respect to the particle's potential sheath is that $(c / a)^{2} \gg\left(1+e \phi_{a} / k T\right)$. When this holds, the fluxes are

$$
\begin{aligned}
& \Gamma_{n_{e}}^{+}=\frac{1}{4} n_{E}\left(8 k T_{p} / \pi m_{e}\right)^{\frac{1}{2} e e^{-\epsilon \phi_{a} / k T_{p}}}\left[1+\left(e \phi_{a} / k T_{p}\right)\right], \\
& \Gamma_{n_{e}}^{-}=\frac{1}{4} n_{e b}\left(8 k T_{e} / \pi m_{e}\right)^{\frac{1}{2}}\left[1+\left(e \phi_{a} / k T_{e}\right)\right] \\
& \Gamma_{H I_{e}}^{+}=2 k T_{p} \Gamma_{n_{e}}^{+} \frac{\left(1+e \phi_{a} / 2 k T_{p}\right)}{\left(1+e \phi_{a} / k T_{p}\right)} \\
& \Gamma_{H_{e}}^{-}=2 k T_{e} \Gamma_{n_{e}}^{-} \frac{\left(1+e \phi_{a} / 2 k T_{e}\right)}{\left(1+e \phi_{a} / k T_{e}\right)}
\end{aligned}
$$

\section{PARTICLE AND GAS IONIZATIONS OF COMPARABLE MAGNITUDE}

The first interaction between the gas and particle ionizations, which is encountered as the level of gas ionization is increased, is the direct suppression of one form of ionization due to the presence of the other. This suppression will be demonstrated for the two cases of a slightly ionized gas and a fully ionized gas, in both of which the plasma is assumed to be in thermodynamic equilibrium.

Saha's equation for gas ionization can be written as

$$
\left(n_{e b} n_{i b}\right) /\left(n_{0}-n_{i b}\right)=K_{Q}(T),
$$

${ }_{12}$ L. Spitzer, Jr., Astrophys. J. 93, 369 (1944).

13 L. Spitzer, Jr., Astrophys. J. 107, 6 (1948).

${ }_{14}$ M. S. Sodha, J. Appl. Phys. 32, 2059 (1961).

15 M. S. Sodha, Brit. J. Appl. Phys. 14, 172 (1963). 
where

$$
K_{G}(T)=2\left(2 \pi m_{e} l k T / h^{2}\right)^{\frac{3}{2}}\left(g_{i} / g_{0}\right) e^{-V / k T},
$$

$h$ is Planck's constant, and $g_{i}$ and $g_{0}$ are the statistical weights of the ion and ground state atom, respectively. For simplicity, it has been assumed that only one of the heavy species is capable of ionization. The density before ionization of this species is $n_{0}$ and its ionization potential is $V$. The requirement that the net current to a particle be zero is given by $\Gamma_{n_{e}}^{-}=\Gamma_{n_{e}}^{+}$, where the ion flux to the particle and the ion flux resulting from surface ionization reactions have been neglected. These fluxes are at least $\left(m_{e} / m_{i}\right)^{\frac{1}{2}}$ smaller than the electron fluxes when the particlo and gas ionizations are of comparable magnitude.

For a slightly ionized gas and a positive particle potential, the above relations combine to yield

$$
\rho^{2}+\rho\left(N / P n_{E}\right) \rho \ln \rho=n_{0} K_{G} / n_{E}^{2},
$$

where $\rho=n_{e b} / n_{E}$. In order to better visualize the direct interaction which occurs between the two types of ionization, two characteristic values of the relative electron density $\rho_{p}$ and $\rho_{G}$ are defined: when the particle density is zero, so that all of the free clectrons are due only to gaseous ionization $\rho_{G}^{2}=n_{0} K_{G} / n_{E}^{2}$; when the density of ionizable gas is zero, so that all the free electrons are from the particles, $\left(1 / \rho_{p}\right) \ln \left(1 / \rho_{p}\right)=P n_{E} / N$. In terms of these two characteristic qualities, $\rho$ is given by

$$
\rho^{2}+\rho \rho_{p}\left[\ln \rho / \ln \left(1 / \rho_{p}\right)\right]=\rho_{G}^{2} .
$$

The anticipated effect, the suppression of one form of ionization due to the presence of the other so that $\rho$ is always less than $\left(\rho_{p}+\rho_{\theta}\right)$, is illustrated in Fig. 4.

For the case of a fully ionized gas, the equation which defines $\rho$ is

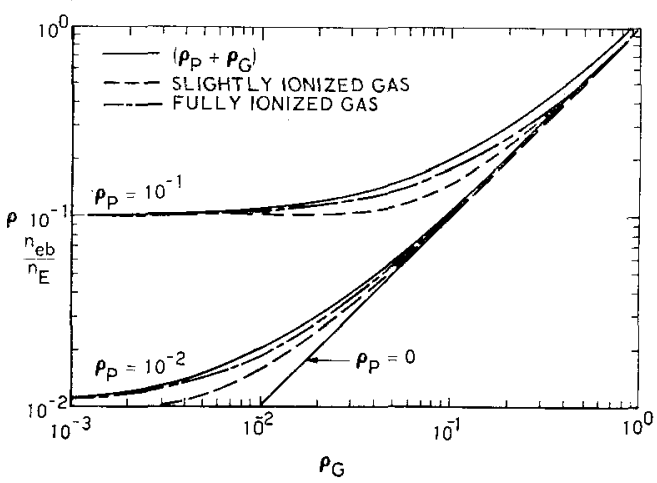

FIG. 4. Total relative electron density versus the relative electron density due to gas ionization alone.

$$
\rho+\rho_{p}\left[\ln \rho / \ln \left(1 / \rho_{p}\right)\right]=\rho_{G},
$$

where $\rho_{p}$ is still defined as above but $\rho_{G}=n_{0} / n_{E}$. In the case of a slightly ionized gas, there were two degrees of freedom; both the gas and particle ionizations were variable. When the gas is fully ionized, there exists only one degree of freedom and, as is also shown in Fig. 4, the total suppression of the ionization is not as large. A similar conclusion has been reached in Ref. 5 .

\section{IONIZATION ENHANCEMENT}

A second interaction between the two types of ionization exists which, although more indirect than the one just considered, is able to produce an electron density in the gas-particle plasma that is several orders of magnitude larger than that which would be expected from either particle or gas ionization alone. This second phenomenon, gaseous ionization enhancement, is now analyzed and a numerical example considered.

\section{A. Formulation}

When hot particles are in a relatively cool but ionizable background gas, the possibility exists that the net energy flux in the electrons emitted from and returning to the particles is sufficient to maintain the temperature of the electron gas outside the particles at a value significantly greater than the temperature of the heavy species and thereby enhance the gaseous ionization. When elastic collisions with the heavy species constitute the major power loss from the electron gas, the relatively large differences in the electron and atomic masses will effectively insulate the electrons from the heavy species and the electron temperature can be almost equivalent to that of the particles. The role of the hot particles in elevating the electron temperature in this situation is similar to that of an electric field which gives rise to nonequilibrium conductivity in a gaseous plasma. ${ }^{16-19}$ Both the hot particles and the electric field provide a power input to the electron gas which is balanced by the power lost to the heavy species through collisions.

In analyzing this phenomenon, several additional simplifying assumptions which can be made are:

(1) Quasi-steady state. The concept of a static

${ }^{16} \mathrm{~J}$. S. Kerrebrock, in Engineering Aspects of Magnetohydrodynamics (Columbia University Press, New York, 1962), p. 327. (1964)

Kerrebrock and M. A. Hoffman, AIAA J. 2, 1080

${ }^{18}$ E. E. Zukoski, T. A. Cool, and E. G. Gibson, AIAA J. 2, $1410(1964)$.

${ }_{19}$ E. E. Zukoski and T. A. Cool, AIAA J. 3, 370 (1965). 
system with three distinct temperatures, particle, gas, and electron, is a realistic one for situations in which the characteristic times for the particle or gas temperature changes are long compared to the gaseous ionization relaxation time and the characteristic time for the establishment of a static charge distribution; the latter characteristic time is usually many orders of magnitude smaller than other times of interest.

(2) Uniform Maxwellian electron temperature. The assumption is made that the energy distribution of the electron gas is Maxwellian and that the electron temperature is spatially uniform. The latter assumption is good in most cases since the electronelectron thermal equilibration length is usually of the same magnitude or much larger than the interparticle distance.

(3) Ionization equilibrium. The assumption is made that at $r=b$ the ionization of the heavy species is in a state of equilibrium which corresponds to the electron temperature. However, since the charge densities and fields do not change appreciably from their values at $r=b$, except in regions on the order of $a$ close to a particle, the assumption of ionization equilibrium is good over the main body of the plasma.

The assumption of equilibrium ionization of the gas at the electron temperature requires that the electron density be high enough so that the electron generation and removal processes are dominated by electron collisions. That is, radiative processes or processes which involve collisions of only heavy species must be unimportant relative to those collisional processes which involve electrons and heavy species. Since both the electron-heavy species inelastic cross sections and the electron mean speed are relatively large, this is a reasonable assumption for many situations.

(4) Electron energy loss. It is assumed that the dominant power loss from the electron gas is through collisions with heavy species. Hence, the power loss of the electron gas per unit volume, which is balanced by the net energy input from the particles, is

$$
\left(\frac{3}{2} k\right)\left(T_{e}-T_{G}\right)\left(n_{e b}\right)\left(8 k T_{e} / \pi m_{e}\right)^{\frac{1}{2}}\left[\overline{\left(m_{e} / m_{G}\right)\left(\delta / \lambda_{e G}\right)}\right]
$$

where

$$
\overline{\left(\frac{m_{e}}{m_{G}}\right)\left(\frac{\delta}{\lambda_{e G}}\right)}=\frac{m_{e}}{2} \sum_{i}\left[\left(\frac{\delta_{j} n_{i}}{m_{i}}\right) \int_{0}^{\infty} Q_{e j}(\epsilon) \epsilon^{2} e^{-\epsilon} d \epsilon\right]
$$

in which $\delta_{j}$ is an energy transfer coefficient of species $j$ (shortly to be discussed), $\lambda_{e G}$ is the electron- heavy species mean free path, $n_{j}$ and $m_{i}$ are the number density and mass of species $j$, respectively, and $\epsilon$ is the normalized electron energy $m_{e} v_{e}^{2} /\left(2 k T_{e}\right)$.

The energy transfer coefficient $\delta_{j}$ is an approximate way to include losses due to electron-heavy species inelastic collisions. If the collisions are elastic, $\delta_{j}$ assumes the value of $\frac{8}{3}$. When rotational, vibrational, and electronic states of a heavy species can be excited by electron impact, values of 10 or 100 for $\delta_{i}$ are not uncommon. Because of its simplicity, accounting for the elcctron energy loss in this way is preferred in this analysis, even though the more rigorous way is to include terms corresponding to each of the energy loss mechanisms.

The elevation of the electron temperature above that of the gas and the accompanying enhancement of gaseous ionization has recently been experimentally demonstrated. ${ }^{18,19}$ In this experiment, a dc electric field supplied the power input to the electron gas and excellont agreement was obtained between the results and the theory which utilized many of the assumptions employed here.

The number of relationships which define the electron density in the plasma can now be reduced to two. The first is Saha's equation, Eq. (20), in which the gas is assumed to be slightly ionized, and the second results when the requirement of steady state is combined with the energy balance on the electron gas. In the requirement of steady state, the particle potential is assumed to be negative, the electron fluxes to and from a particle are equated, and the ion fluxes are neglected. The powcr balance on the electron gas consists of the equation of the power lost by collisions with the heavy species, expression (24) to the power gained by thermal replenishment from the particles, which is found from Eqs. (14) and (15). The result of this balance, when combined with the equation of steady state, yields

$$
\left(\frac{T_{p}-T_{e}}{T_{e}-T_{G}}\right)\left(\frac{T_{p}}{T_{e}}\right)^{\frac{1}{2}}=\frac{3}{4 \pi a^{2} N}\left[\overline{\left(\frac{m_{e}}{m_{G}}\right)\left(\frac{\delta}{\lambda_{e G}}\right)}\right]\left(\frac{n_{e b}}{n_{E}}\right) .
$$

If the right-hand side of Eq. (25) is very much less than unity, $T_{e}$ will be close to $T_{p}$. This is a very possible situation in view of the relative magnitudes of the electron and atomic masses. The transition of the temperature of the electrons from the gas temperature to the particle temperature is shown in Fig. 5, where $T_{e} / T_{G}$ is plotted as a function of the right-hand side of Eq. (25) for values of $T_{p} / T_{G}$ between 1.0 and 2.0. Generally, both Eqs. (20) and (25) must be solved simultaneously for $T_{e}$ and $n_{e b}$. 


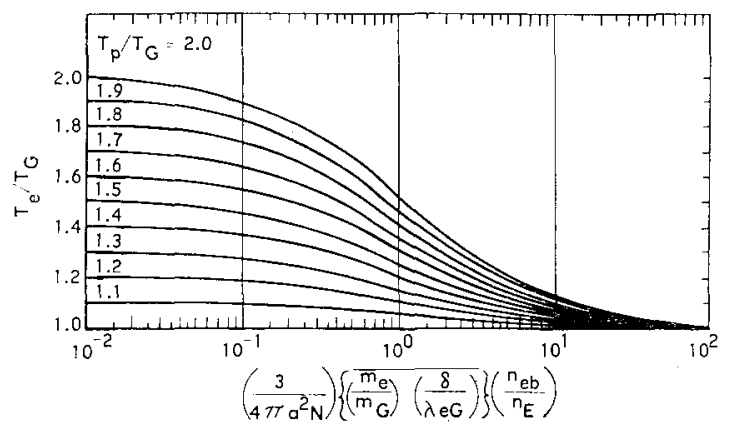

FIG. 5. Electron temperature divided by the gas temperature versus the relative strength of the electron-heavy gas species collision loss.

\section{B. Ionization Enhancement in the Exhaust Plume of a Solid Rocket Motor}

The numerical example of gaseous ionization enhancement which is considered illustrates the magnitude of the enhancement that can occur in the cxhaust plume of a solid motor under conditions such that the assumptions employed here are valid. The combustion products in the plume may contain both thermionically emitting alumina particles, whose temperature change lags that of the cooling gas, ${ }^{20}$ and alkali metal impurities. ${ }^{1}$ The observed electron densities are usually on the order $10^{10}-10^{11} \mathrm{~cm}^{-3}$, but equilibrium calculations of the particle ionization at the particle temperature or the alkali metal ionization at the gas temperature yield considerably lower values. One possibility is that the chemical reactions, including recombination of electrons and ions, become frozen upstream of the exit plane and thereby produce electron densities which are higher than the equilibrium values. A second possibility, and one which can be attributed directly to the presence of the particles, is the type of gaseous ionization enhancement studied here.

Some of the parameters which are used to describe

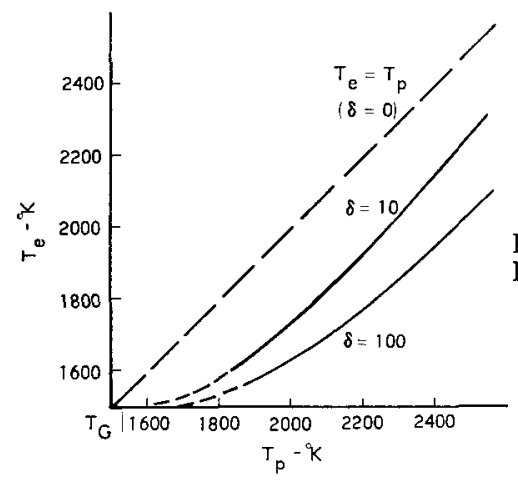

FIG. 6. Electron temperature versus the particle temperature.

${ }^{20}$ D. J. Carlson, Am. Rocket Soc. J. 34, 1107 (1963).

the conditions in the plasma have been chosen in an arbitrary but, it is hoped, realistic manner. Others have been taken directly from Ref. 1 . The average properties of the gas are taken to be $n_{G}=10^{17} \mathrm{~cm}^{-3}$, molecular weight $27, T_{G}=1500^{\circ} \mathrm{K}, 0 \leq \delta \leq 10^{2}$, and electron-gas total collision cross section = $10^{-15} \mathrm{~cm}^{2}$. The alkali metal impurity is assumed to be 300 ppm of potassium vapor. The parameters of the alumina particles are taken to be $N=10^{7} \mathrm{~cm}^{-3}$, $a=10^{-4} \mathrm{~cm}, e \Phi=3.77 \mathrm{eV}$, and $\kappa=1.17 \times 10^{-2}$.

Equations (20) and (25) have been used to calculate $T_{e}$ and $n_{e b}$. The results are shown in Figs. 6 and 7 . When $T_{p}$ is close to $T_{G}, n_{E}$ is very much less than $n_{e b}$ so that the power input to the electrons is not sufficient to displace them far from thermal equilibrium with the gas. However, it can be shown that since $\frac{1}{2} V<e \Phi$, increases made in $T_{p}$ will tend to drive $T_{p} / T_{\text {e }}$ closer to unity at the higher values of $T_{p}$. From Fig. 7, it is seen that for $\delta=10$ and a particle temperature lag of $500^{\circ} \mathrm{C}$, the ionization is enhanced by a factor of 10 . A lag of $800^{\circ} \mathrm{C}$ gives a factor of enhancement of $10^{2}$ or an electron density of $3.5 \times 10^{11} \mathrm{~cm}^{-3}$. Thus, even though the gas temperature drops rapidly in this system, the particles are able to keep the electron temperature relatively high and thereby enhance the ionization.

It is of importance to point out that the above treatment actually is an underestimate of the magnitude of the enhancement because of the neglect of surface ionization. When the particle temperature is higher than that of the electrons, the rate of surface ionization will exceed the rate of surface recombination and a direct contribution to the electron density results. Also, since the recombination rate must then exceed the ionization rate in the gas, a second process exists by which energy is

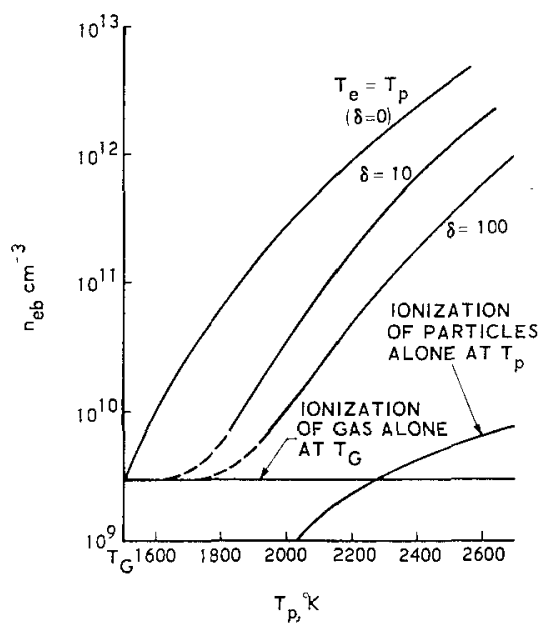

FIG. 7. Electron density versus the particle temperature. 
added to the electron gas, the temperature elevated and the ionization enhanced.

An inverse to the above process is also possible. If the particle temperature is lower than that of the gas, the ionization would be reduced. The electron temperature reduction would be of importance to the point where the collisions between electrons and heavy species no longer constituted the dominant electron generation and removal process. Surface recombination would exceed surface ionization and, besides a decrease in the electron temperature, a direct reduction in the electron density would result. If the particle temperature were cool enough, thermionic emission would become negligible and the particles would also reduce the ionization directly by electron absorption.

\section{EFFECT OF PARTICLES ON IONIZATION RELAXATION TIMES}

In addition to being capable of appreciably changing the steady-state electron density in a plasma, the presence of particles can also significantly alter the time rates of change of the electron density in nonsteady situations. A particle can act either as an electron source, through thermionic emission or surface ionization, or as an electron sink, through the combined effects of electron absorption and surface recombination. Under some conditions in a transient process, the particles can be the major electron source or sink and thereby determine the time rate of change of the clectron density.

In order to demonstrate that the effect of the particles on the ionization relaxation times can be large, the characteristic time for electron removal is computed. It is assumed that the electron mean free path is much larger than the interparticle distance, that all the electrons which strike a particle are either absorbed or recombine at its surface, and that the particles are relatively cool so that electron addition by the particles is negligible. Thus, the rate of electron removal is

$$
\dot{n}_{e b}=-\pi a^{2} N n_{e b} \bar{C}_{e} e^{e \phi_{a} / k T_{e}},
$$

where $\phi_{a}<0$ and $\vec{c}_{e}$ is the electron mean thermal speed. Defining the characteristic time for electron removal as

$$
\tau_{E R}=-n_{e b} / \dot{n}_{e b}
$$

it is seen that

$$
\tau_{E R}=\left(\pi a^{2} N \bar{c}_{e} e^{e \alpha_{a} / k T_{\epsilon}}\right)^{-1} .
$$

Using $a=10^{-6} \mathrm{~cm}, N=10^{10} \mathrm{~cm}^{-3}, T_{e}=1000^{\circ} \mathrm{K}$ and $\exp \left(e \phi_{a} / k T\right) \simeq 1, \tau_{E R}$ is $2 \times 10^{-6} \mathrm{sec}$ which is usually much shorter than the corresponding time for gaseous ionization relaxation.

This electron removal process could be of importance in flows in which the gas and particle temperatures drop rapidly and the degree of gaseous ionization becomes frozen. If the value of $\tau_{E R}$ is much smaller than the characteristic time for gaseous ionization relaxation, the electron density would be controlled by the particle electron removal process and could be reduced well below the gaseous frozen state value. This is a possible means of electron removal in the downstream region of the wake of a high-altitude re-entry body or the exhaust plume of a solid rocket nozzle.

\section{CONCLUSIONS}

The preceding analysis has shown that the presence of particles in a gascous plasma is able to change the value of the elcctron density appreciably from the value it would assume if the particles were not present. There exists a variety of electron-particle interactions in steady and nonsteady situations which can be used both to predict and to control the electron density of a gas-particle plasma.

\section{ACKNOWLEDGMENTS}

It is a pleasure for the author to express his appreciation to Dr. Edward Zukoski for many stimulating discussions during this investigation. Gratitude is also expressed to Dr. Frank Marble, who introduced the author to the field and has given invaluable advice throughout the progress of the work. Dr. Stanley Byron is thanked for some enlightening discussions on some of the latter points in the paper.

This research was carried out at the Daniel and Florence Guggenheim Jet Propulsion Center at the California Institute of Technology and supported by the Air Force Office of Scientific Research under Grant AF-AFOSR-160-63. The paper was written while the author was at the Research Laboratories of the Philco Corporation, Newport Beach, California. 